\title{
Cerebral Blood Flow Measurement in the Assessment of Post-Traumatic Cerebral Contusions
}

\author{
P. Pifarré ${ }^{1}$, G. Cuberas ${ }^{1}$, B. Benejam ${ }^{2}$, L. Frascherrii ${ }^{1}$, J. Sahuquillo ${ }^{2}$, J. Castell-Conesa ${ }^{1}$ \\ ${ }^{1}$ Nuclear Medicine Department, Hospital Universitaria de la Vall d'Hebron, Universitat Autònoma de Barcelona, \\ Barcelona, Spain \\ ${ }^{2}$ Neurosurgery Department, Hospital Universitari de la Vall d'Hebron, Barcelona, Spain \\ ${ }^{3}$ Radiology Department, Hospital Universitari de la Vall d'Hebron, Barcelona, Spain \\ E-mail: paloma.pifarre@crccorp.es \\ Received September 22, 2011; revised October 20, 2011; accepted November 8, 2011
}

\begin{abstract}
Brain trauma (BT) is extremely common in the Western society, and has been identified as the main cause of death and disability in the under-40 age group. Many aspects of the pathophysiological mechanisms involved and the effect of changes in cerebral metabolism are unclear. The aim of this study was to establish the relationship between anatomical changes and deranged cerebral perfusion in patients with cerebral contusions, using Computed Tomography (CT) and Single Proton Emission Computed Tomography (SPECT). Twentytwo (22) patients who had suffered BT were recruited. All patients underwent SPECT and CT head scans on the same day. 18 were men. Patient average age was 45.6. Patients were assessed using the Glasgow scale (average 10.6). Cause of trauma included traffic accidents (9 patients) and falls (13 patients). A 4-slice spiral CT scan was performed. For each contusion, areas of bleeding, edema, and healthy perilesional tissue were distinguished. SPECT was performed with $20 \mathrm{mCi}$ of 99 mTcECD using a dual-head gamma camera (128 $\times$ 128 matrix). CT scan revealed a single lesion in 12 patients, and more than one lesion in 10 . The biggest lesions found on CT were located in the frontal region in 13 patients; temporal region in 4; and parietal region in 1; four patients had poorly defined lesions. A total absence of perfusion was visible in 18 patients in the hemorrhagic area and in 14 patients in the edema, In 7 cases SPECT showed hypoperfusion that did not correspond to any morphological changes on the CT scan. Quantitative of fused lesions appearing on both CT scan and SPECT revealed severe perfusion defects in the hemorrhagic area (17.8\%) and in the edema (29.4\%). In our study, regional cerebral blood flow adds relevant information on encephalic damage in patients with BT.
\end{abstract}

Keywords: Brain Trauma, SPECT, CT, Cerebral Blood Flow

\section{Introduction}

Brain trauma (BT) has been called the silent epidemic of the 21st century. The incidence in Western countries is 200 - 400 in every 100,000 inhabitants/year, and HI (Head Injury) are the foremost cause of death and disability in the under 40 age group [1,2]. The findings of a number of multicenter studies performed in recent years have shown that mortality rates in general hospitals are high, generally ranging between $30 \%$ and $40 \%$. Neuroprotective mechanisms employed following trauma are often less than ideal [3], and neurocritical patient management tends to focus on reducing or preventing cere- bral hypoxia [4]. When metabolic derangements arising from BT are interpreted it is difficult to determine the extent and severity of ischemia and cell necrosis and the significance of the edema $[5,6]$.

Brain trauma (BT) is defined as a physical lesion or functional deterioration of cranial content owing to an abrupt mechanical energy exchange [7]. Cerebral contusions are focal lesions in which there are delimited areas of bleeding, edema, tissue necrosis and destruction of the central nervous system. Although the severity of the biomechanical impact is important from the standpoint of survival and functional outcome, systemic or intracranial aggressions that appear minutes, hours or days after ac- 
tual trauma occured are responsible for the worsening condition of many patients presenting with BT [8]. This implies that the pathophysiological mechanisms that contribute to neuronal injury and affect patients' clinical evolution need to be identified and remedied.

Following trauma the brain has both a greater chance of experiencing ischemia and decreased tolerance to hypoxia. The appearance of hypoxia or ischemia following BT is currently attributed to vascular factors that act by reducing or eliminating the compensatory mechanisms of cerebral vessels in response to an insult [9]. One of the first mechanisms that is invariably affected in BT is the balancing of metabolic demands and substrate supply from cerebral blood flow (CBF), and it is clear that a large percentage of patients show a reduction of CBF in the first hours following BT with respect to the oxygen metabolism rate [10]. Nevertheless, it continues to be difficult to know how and when ischemia occurs or the degree and significance of hypoperfusion, since the effect of trauma on cerebral circulation and metabolism is temporary and spatially variable $[5,6]$, and CBF in these patients fluctuates. In a single patient, areas with decreased cerebral flow can coexist with areas of increased perfusion [11,12].

Although ischemic areas tend to be found in regions around the trauma lesion [13], they can also be found in normal brain structures [14], and methods are needed to determine the borderline between hypoperfusion and ischemia in contusions. In other words, tissues affected by hypoperfusion should be defined using areas based not on spatial contiguity, but on pathophysiological contiguity.

Cerebral SPECT (Single Photon Emission Computed Tomography) is a tomographic detection technique providing 3-D information on cerebral perfusion and metabolism. Although the information obtained constitutes an adjunct to observations made based on neuroanatomical techniques, the findings can be different from the structural changes seen with conventional techniques, and provide further information determining the presence or absence of live tissue. Uptake of perfusion tracers requires the existence of viable neuron cells, suggesting that they are better able to reveal the extent of irreversible brain lesions than anatomical images alone. Furthermore, the regions adjacent to the contusion corresponding to edema and ischemic penumbra might be defined through a quantitative uptake assessment with SPECT.

The aim of our study was to determine the relationship between different neuroimaging patterns, SPECT and CT (computerized tomography), in assessing the extent and severity of cerebral perfusion changes in cerebral contusions seen in patients with $\mathrm{HI}$ and determining the viability of involved brain tissue. For this purpose, a method- ology for multimodal recording and quantification of perfusion defects was evaluated.

\section{Materials and Method}

\subsection{Patients}

Between January 2005 and September 2006, a prospective assessment was done of a total of 22 consecutive patients (6 women) average age 45.6 (range: 16 - 75) who were admitted to the Neurotraumatology Service of our hospital following BT. All patients presented posttraumatic cerebral contusions. However, only those that did not require immediate surgical evacuation were included in the study. All were treated in accordance with the protocol for patients with BT in the guidelines published and updated by the Brain Trauma Foundation [15]. A relative of every patient signed informed consent for the study under a protocol approved by our institution.

The causes of $\mathrm{HI}$ among the patients in the study were an accidental fall (40.9\%), traffic accident (22.7\%) fall from a height (18.2\%), being struck by a vehicle (18.2\%). In the initial neurological assessment, 31.8\% of patients had severe BT (GCS < 8), 36.4\% had moderate BT (GCS between 8 and 13) and 36.4\% had mild BT (GCS > 13).

\subsection{Methodology}

a) Study sequence

Cerebral SPECT and CT scan were performed on the 22 patients, all with recent trauma, 24 - 48 hours after BT (average, 39.5 hours). CT and SPECT were performed the same day, to get images that were as similar as possible.

b) Acquisition features

CT scan: a 4-slice spiral CT scan was performed (Siemens Somatom DHR, Siemens Medical Systems inc., Erlangen, Germany) aligning the gantry with the orbitomeatal line to obtain $10 \mathrm{~mm}$ thick slices of the posterior fossa, and $5 \mathrm{~mm}$ slices of the supratentorial compartment.

Perfusion SPECT: A $20 \mathrm{mCi}$ dose of ECD (Neurolite, Bristol Myers Squibb) was administered intravenously. SPECT images were acquired 20 - 30 minutes following injection of the tracer, using a dual-head Siemens e.cam gamma camera and parallel LE HR Siemens collimators, with an elliptical orbit of $360^{\circ}$. Images were obtained every 3 degrees (20 seconds/frame) with a $128 \times 128$ pixel matrix, adjusting the $99 \mathrm{mTc}$ photopeak with a $15 \%$ window. Frames were reconstructed using filtered back projection (Butterworth filter, 5/0.4) with compensation for scatter (Chang $\mu=0.11 / \mathrm{cm}$. Slices were obtained on the transversal, coronal and sagittal planes. Any radiation 
protection measures were given because it was a routinary clinical study.

c) Image analysis

The tomographic images were analyzed independently, qualitatively and quantitatively, by two experienced blinded nuclear physicians, who in each case had access to the clinical information and other tests that had been performed.

\subsection{Qualitative Analysis}

This analysis consisted of a global assessment of cerebral perfusion/metabolism analyzing cortical and subcortical regions and the cerebellum. Uptake was assessed in three grades: normal, hypoperfusion and no uptake [16] by two experienced observers and discrepancies were solved by consensus.

\subsection{Quantitative Analysis}

The algorithms used in the co-registration process and in the quantitative analysis, were developed in Gregorio Marañon Hospital Image Laboratory, which was in collaboration with our department [29,30]. Images from both studies (anatomical and functional) were exported in DICOM format on a PC (intel Pentium 4 with Windows XP). The quantitative analysis was divided into 2 parts. First, a global analysis of total volume of interest was performed to determine global perfusion in the regions being studied. Subsequently, a small region of interest in the center of the injured area was selected to avoid scatter to healthy tissue, due to partial volume effect.

1) Selection of volumes of interest (VOI): CT frames were used to select slices with visible lesions, and regions of interest (ROI) were delimited for subsequent analysis. For each contusion the lesion core and perilesional edema (ischemic penumbra) were defined. (Figure 1).

To improve reproducibility a semi-automatic method was used based on the "seeded region growing algorithm”. This algorithm involves clustering in a region of interest all interconnected (i.e., adjacent) pixels, starting from a seed inserted by the user into the region to be analyzed. Starting with the pixel that has been seeded, the region grows, adding to the region of interest all those adjacent pixels with values included in the range selected by the user and leaving out all those above or below the upper and lower limits selected. It was discovered that values in Hounsfield Units (HU) at the lesion core varied between 50 and 75, and values for edema were in the range of 10 - $25 \mathrm{HU}$. An ROI was delimited on the cerebellum. Delimitation was done for both hemi-

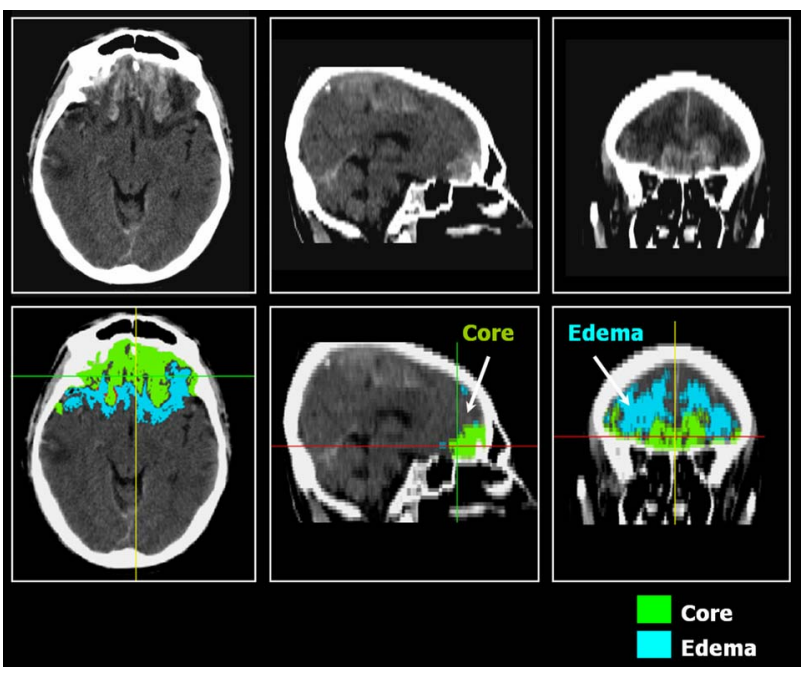

Figure 1. Patient affected of bilateral frontal lesion. We delimited volumes of interest in core and in edema on CT enhanced scan.

spheres, except in cases of cerebellar diaschysis when it was only done for the "normal" hemisphere.

To determine lesion volume, the cubic centimeters of each of the previously measured volumes of interest were calculated. The co-registration algorithm applied between SPECT and CT was a rigid register based on mutual information. Co-register of SPECT and CT images ensured that they coincided anatomically. Subsequently, image fusion was done to check the result in three spatial dimensions.

To determine the extent of involvement based on cerebral perfusion in the areas of interest, the ROIs defined above on CT were used and an average was obtained for absolute counts for the same regions on SPECT slices. To quantify the degree of perfusion in both regions of interest (lesion core and edema), the edema/ cerebellum and core/cerebellum ratios were calculated (Figure 1). Penumbra area was defined in the CT image and, to quantify brain perfusion, this region of interest was moved into the co-registered SPECT image and mean counts were extracted.

Selection of regions of interest (ROI): It was observed that in pixels adjacent to healthy tissue, or in cases where the lesion was very small, the values obtained using VOIs were contaminated due to the partial volume effect, and consequently the value for cerebral perfusion in that region was overestimated. A second analysis was therefore performed to obtain values that more closely reflected the reality. A small region of interest at the geometric center of the lesion volume was taken, with the aim of finding values with less of a bias due to the partial volume effect.

d) Statistical analysis 
Variables were analyzed to determine whether their distribution was normal, using a Kolmogorov-Smirnof test. After determining that they all fitted a Gaussian field model, a means comparison test (T-Test) was performed with variable pairs.

To assess the degree of uptake in the edema and the core and in order to classify the quantitative values, a distribution of mean was done and it was observed that there were 3 main classes. For this reason, patients were divided according to the intensity of perfusion defects into three categories: group 1 if uptake was below 45\%; group 2 if uptake was between $45 \%$ and 55\%; and group 3 if uptake was over $55 \%$.

\section{Results}

Twenty-two cerebral SPECT scans were performed. Cerebral perfusion seen in all of these studies was generally considered pathological.

\subsection{Anatomical Assessment}

CT revealed a single lesion in 12 patients (54.5\%), and more than one lesion in 10 patients (45.5\%).

Lesions were located in the following regions: left frontal $(\mathrm{n}=4$; $18.2 \%)$; right frontal $(\mathrm{n}=6$; $27.3 \%)$; bifrontal ( $\mathrm{n}=3$; 3.6\%); left temporal $(\mathrm{n}=2$; $9 \%)$; right temporal ( $\mathrm{n}=2$; $9 \%$ ); right parietal ( $\mathrm{n}=1 ; 4.5 \%)$; and poorly defined lesions ( $n=2 ; 9 \%)$. Perfusion derangements in the frontal region clearly predominated (58\%).

\subsection{Qualitative SPECT Assessment}

Tracer uptake in the lesion core and the perilesional area was defined. None of the patients had tracer uptake in the normal range in these areas. In the lesion core, 18 patients (82\%) showed no tracer uptake while (18\%), had hypoperfusion. In the perilesional region no uptake was seen in 14 patients (64\%), and hypoperfusion was seen in 8 (36\%). In 12 patients changes in cerebral perfusion were observed in more than one location. Of these changes, 4 were regions with hypo-perfusion, of a smaller size than the main lesion and separated from it by normal brain tissue. In three cases, the hypoperfused area was located in the cerebral parenchyma directly opposite the initial lesion (Figure 2). Hypoperfusion was seen in 7 SPECT cases on SPECT but this did not correspond to any morphological change on CT.

\subsection{Quantitative SPECT Assessment}

Statistically significant differences were seen between edema and lesion core, yielding $\mathrm{p}=0.008$ when the vari-

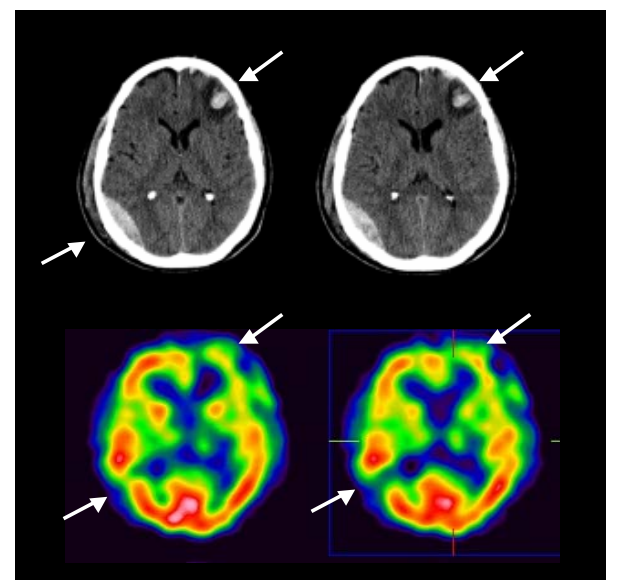

Figure 2. CT and SPECT perfusion imaging of a patient with left frontal primarily brain trauma. In the hemorrhagic lesion seen on the $\mathrm{CT}$ in the right posterior temporal region, SPECT images show an absence of perfusion.

ables reflecting the number of counts in the entire volume of the lesion (VOI) were analyzed, and $\mathrm{p}=0.003$ when counts in the geometric center of the lesion (ROI) were analyzed. Data distribution is shown in Figure 3.

Means, standard deviations and ranges of values for both analyses in the two regions appear in Table 1. The for minimum uptake value obtained for perfusion in the areas of edema and core in the VOI analysis was found to be higher than the value obtained for the ROI.

For edema, it was observed that with VOI analysis, $36.4 \%$ of patients were in group $1,31.8 \%$ of patients in group 2, and the remainder, in group 3 . Taking ROI minimum value, $81.8 \%$ of patients were classified as group $1,18.2 \%$ were in group 2 , and no patients had uptake levels that would include them in group 3 .

Table 1. Means, standard deviations and ranges of values obtained for VOI and ROI analyses in core and edema regions.

\begin{tabular}{ccc}
\hline \multicolumn{3}{c}{ \% Perfussion } \\
& Edema & Core \\
Media & 47.9 & 37.1 \\
DE & 10.7 & 10.5 \\
min & 14.1 & 19.2 \\
max & 60.3 & 57.9 \\
& & ROI \\
Media & Edema & Core \\
DE & 29.4 & 17.8 \\
min & 13.5 & 11.2 \\
max & 7.7 & 3.2 \\
\hline
\end{tabular}




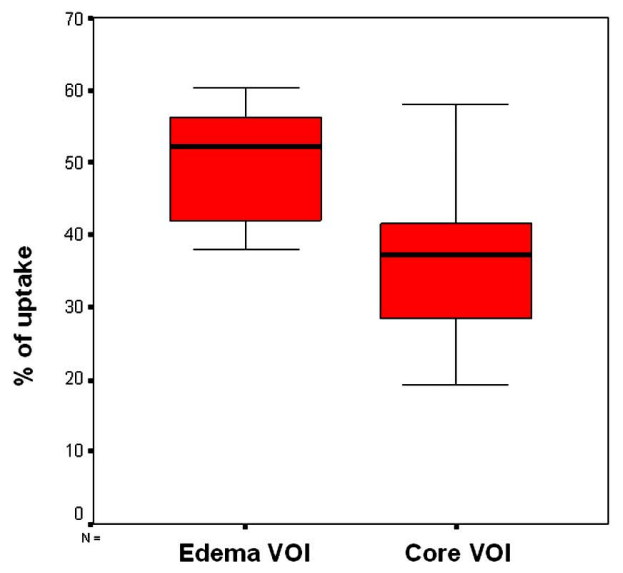

(a)

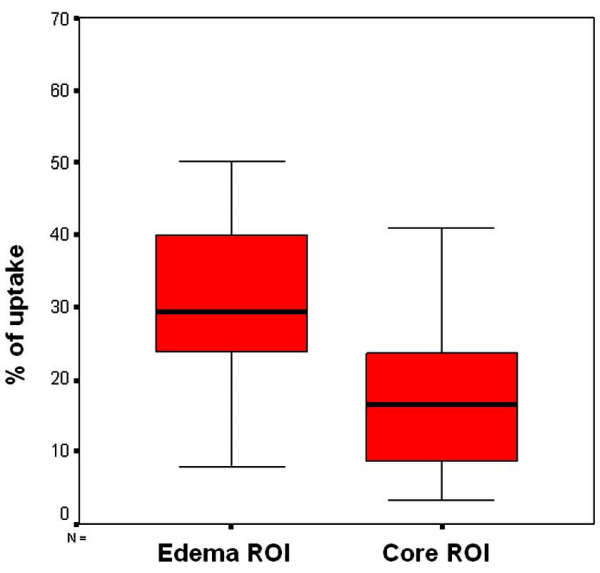

(b)

Figure 3. (a) Distribution of the number of counts in the entire lesion volume (VOI); (b) Distribution of the number of counts in the geometric center of the lesion (ROI).

When core uptake was analyzed it was seen that using VOI analysis, $77.3 \%$ of patients were in group 1 , whereas $18.2 \%$ were in group 2 , and $4.5 \%$ in group 3 . Using ROI analysis, $100 \%$ of patients had uptake levels placing them in group 1.

\section{Discussion}

Scientific evidence exists that in cerebral contusions, injured areas can have different profiles in terms of metabolism and regional cerebral flow (core, perilesional area, etc). There is also a hypothesis that the lesion core behaves like an area of tissue necrosis whose metabolic profiles are incompatible with the survival of brain tissue, and that the deterioration of areas around the lesion (ischemic penumbra) precedes neurological deterioration in those patients, because such areas are unstable both in time and space, and if a state of ischemia is prolonged over time, even without a greater reduction in CBF, cell death may eventually ensue [17-20].

In our study we analyzed 22 patients with BT who underwent cerebral perfusion SPECT and cranial CT scan.

In various studies it has been observed that cerebral SPECT detects a greater number and degree of cerebral derangements than anatomical techniques such as CT scan $[22,23,28]$.

In their study, Gray et al. [24] showed that $80 \%$ of patients with BT had altered cerebral perfusion, whereas only 55\% had alterations that appeared on CT scan. Similarly, a number of studies have shown that the changes seen in cerebral perfusion can explain sequelae in patients in the absence of morphological abnormalities on CT scan.

In our study we observed that in all patients in whom lesions appeared on CT scan, a matching area of hypoperfusion was seen on SPECT. These lesions were primarily located in the frontal area, the region typically injured in BT due to the gliding effect of the brain under the skull $[25,28]$.

Uptake of perfusion defects in core and edema areas can be obstructed by the partial volume effect. In the ROI analysis, where the geometric center of the lesion is used, this interference did not occur, and the average lesion value was lower. The effect thereof can be more important when analyzing small size traumas, where the entire lesion area can be influenced by activity in adjacent pixels corresponding to normal tissue.

We feel it is important to mention that parenchymatous lesions were larger and more frequent on SPECT than with CT scan. Furthermore, hypoperfusion areas were detected in regions that were ostensibly normal using CT scan, probably due to changes in remote neuronal activity (diaschysis) [23,26].

An important finding of our study was that in all patients both the contusion core region and penumbra area corresponded to an intensely hypoperfused area. Such findings suggest that in most patients these regions have little likelihood of tissue recovery. Notwithstanding, low CBF levels may indicate the existence of ischemia or down regulated metabolic balance [27]. The uptake threshold for the existence of viable tissue has yet to be determined, although to do so will require following the long-term evolution of the metabolic activity of cerebral tissue.

\section{Conclusions}

Cerebral perfusion SPECT permits determination of cerebral flow in areas with direct trauma lesions, and de- 
tection of distant alterations resulting from the existence of secondary trauma or dysafferentation. Combining CT and SPECT assessments makes it possible to determine the extent and severity of cerebral injury in traumatic lesions and determine tissue viability in core, edema and perilesional tissue. Subsequent studies should determine whether these data have prognostic value with regard to the possibility of neurological recovery in patients with BT.

\section{References}

[1] J. Bruns Jr. and W. A. Hauser, "The Epidemiology of Traumatic Brain Injury: A Review,” Epilepsia, Vol. 44, Supplement 10, 2003, pp. 2-10. doi:10.1046/j.1528-1157.44.s10.3.x

[2] F. Tagliaferri, C. Compagnone, M. Korsic, F. Servadei and J. Kraus, “A Systematic Review of Brain Injury Epidemiology in Europe,” Acta Neurochirurgica (Wien), Vol. 148, No. 3, 2006, pp. 255-268. doi:10.1007/s00701-005-0651-y

[3] A. I. Maas, E. W. Steyerberg, G. D. Murray, R. Bullock, A. Baethmann, L. F. Marshall and G. M. Teasdale, "Why Have Recent Trials of Neuroprotective Agents in Head Injury Failed to Show Convincing Efficacy? A Pragmatic Analysis and Theoretical Considerations," Neurosurgery, Vol. 44, No. 6, 1999, pp. 1286-1298. doi:10.1097/00006123-199906000-00076

[4] R. M. Chesnut, "Secondary Brain Insults after Head Injury: Clinical Perspectives,” New horizons Baltimore Md, Vol. 3, No. 3, 1995, pp. 366-375.

[5] G. J. Bouma and J. P. Muizelaar, "Evaluation of Regional Cerebral Blood Flow in Acute Head Injury by Stable Xenon-Enhanced Computerized Tomography,” ACTA Neurochirurgica Supplementum (Wien), Vol. 59, 1993, pp. 34-40.

[6] J. P. Coles, P. S. Minhas, T. D. Fryer, P. Smielewski, F. Aigbirihio, T. Donovan, S. P. Downey, G. Williams, D. Chatfield, J. C. Matthews, A. K. Gupta, T. A. Carpenter, J. C. Clark, J. D. Pickard and D. K. Menon, "Effect of Hyperventilation on Cerebral Blood Flow in Traumatic Head Injury: Clinical Relevance and Monitoring Correlates," Critical Care Medicine, Vol. 30, No. 9, 2002, pp. 1950-1959. doi:10.1097/00003246-200209000-00002

[7] B. Jennett and M. Bond, “Assessment of Outcome after Severe Brain Damage: A Practical Scale,” Lancet, Vol 305, No. 7905, 1975, pp. 480-484. doi:10.1016/S0140-6736(75)92830-5

[8] P. A. Jones, P. J. Andrews, S. Midgley, S. I. Anderson, I. R. Piper, J. L. Tocher, A. M. Housley, J. A. Corrie, J. Slattery and N. M. Dearden, "Measuring the Burden of Secondary Insults in Head-Injured Patients during Intensive care," Journal of Neurosurgical Anesthesiology, Vol. 6, No. 1, 1994, pp. 4-14.

[9] J. Sahuquillo, A. Rodríguez-Baeza, M. Vaguean, F. Reina, L. Campos and E. Rubio, “Autorregulación Cerebral: Conceptos Fisiopatológicos y Metodología para Su Valora- ción en el Paciente Neurotraumatizado,” Medicina Intensiva, Vol. 20, 1996, pp. 69-78.

[10] G. J. Bouma, J. P. Muizelar and S. C. Choi, "Cerebral Circulation and Metabolism after Severe Traumatic Brain Injury: The Elusive Role of Ischaemia,” Journal of Neurosurgery, Vol. 75, No. 5, 1991, pp. 685-693. doi:10.3171/jns.1991.75.5.0685

[11] J. Overgaard and W. A. Tweed, "Cerebral Circulation after Head Injury. Cerebral Blood Flow and Its Regulation after Closed Head Injury with Emphasis on Clinical Correlations," Journal of Neurosurgery, Vol. 41, No. 5, 1974, pp. 531-541. doi:10.3171/jns.1974.41.5.0531

[12] W. D. Obrist, T. W. Langfitt, J. L. Jaggi, J. Cruz and T. A. Gennarelli, "Cerebral Blood Flow and Metabolism in Comatose Patients with Acute Head Injury. Relationship to Intracranial Hypertension," Journal of Neurosurgery, Vol. 61, No. 2, 1984, pp. 241-253. doi:10.3171/jns.1984.61.2.0241

[13] G. J. Bouma, J. P. Muizelaar, W. A. Stringer, S. C. Choi, P. Fatouros and H. F. Young, "Ultra-Early Evaluation of Regional Cerebral Blood Flow in Severely Head-Injured Patients Using Xenon-Enhanced Computerized Tomography,” Journal of Neurosurgery, Vol. 77, No. 3, 1992, pp. 360-368. doi:10.3171/jns.1992.77.3.0360

[14] J. P. Coles, P. S. Minhas, T. D. Fryer, P. Smielewski, F. Aigbirihio, T. Donovan, S. P. Downey, G. Williams, D. Chatfield, J. C. Matthews, A. K. Gupta, T. A. Carpenter, J. C. Clark, J. D. Pickard and D. K. Menon, "Effect of Hyperventilation on Cerebral Blood Flow in Traumatic Head Injury: Clinical Relevance and Monitoring Correlates,” Critical Care Medicine, Vol. 30, No. 9, 2002, pp. 1950-1959. doi:10.1097/00003246-200209000-00002

[15] The Brain Trauma Foundation, “The American Association of Neurological Surgeons. The Joint Section on Neurotrauma and Critical Care. Methodology," Journal of Neurotrauma, Vol. 17, No. 6-7, 2000, pp. 561-562.

[16] H. M. Abdel-Dayem, H. Abu-Judeh, M. Kumar, et al., "SPECT Brain Perfusion Abnormalities in Mild or Moderate Traumatic Brain Injury,” Clinical Nuclear Medicine, Vol. 23, No. 5, 1998, pp. 309-317. doi:10.1097/00003072-199805000-00009

[17] M. Furlan, G. Marchal, F. Viader, J. M. Derlon and J. C. Baron, "Spontaneous Neurological Recovery after Stroke and the Fate of the Ischemic Penumbra," Annals of Neurology, Vol. 40, No. 2, 1996, pp. 216-226. doi:10.1002/ana.410400213

[18] R. Invertí, G. Bellinzona and M. Langer, "Cerebral Tissue PO2 and SjvO2 Changes during Moderate Hyperventilation in Patients with Severe Traumatic Brain Injury," Journal of Neurosurgery, Vol. 96, No. 1, 2002, pp. 97102. doi:10.3171/jns.2002.96.1.0097

[19] G. Marchal, V. Beaudouin, P. Rioux, V. de la Sayette, F. Le Doze and F. Viader, "Prolonged Persistence of Substantial Volumes of Potentially Viable Brain Tissue after Stroke: A Correlative PET-TC Study with Voxel-Based Data Analysis,” Stroke, Vol. 27, No. 4, 1996, pp. 599-606. doi:10.1161/01.STR.27.4.599

[20] W. D. Heiss, L. Kratch, A. Thiel, M. Grond and G. Pa- 
wlik, "Penumbral Probability Thresholds of Cortical Flumazenil Binding and Blood Flor Predicting Tissue Outcome in Patientes with Cerebral Ischaemia," Brain, Vol. 124, No. 1, 2001, pp. 20-29. doi:10.1093/brain/124.1.20

[21] H. M. Abdel-Dayem, H. Abu-Judeh, M. Kumar, et al., "SPECT Brain Perfusion Abnormalities in Mild or Moderate Traumatic Brain Injury,” Clinical Nuclear Medicine, Vol. 23, No. 5, 1998, pp. 309-317. doi:10.1097/00003072-199805000-00009

[22] H. M. Abdel-Dayem, S. A. Sadek and K. Kouris, "Changes in Cerebral Perfusion after Acute Head Injury. Comparison of CT with 99mTc HMPAO SPECT," Radiology, Vol. 165, 1987, pp. 221-226.

[23] J. C. Masdeu, R. L. Van Heertum, A. Kleiman, et al., "Early SPECT in Mild Head Trauma. A Controlled Study," Journal of Neuroimaging, Vol. 4, 1994, pp. 177-181.

[24] B. G. Gray, M. Ichise, D. G. Chung, J. C. Kirsh and W. Franks, "Technetium-99m-HMPAO SPECT in the Evaluation of Patients with a Remote History of Traumatic Brain Injury: A Comparison with X-Ray Computed Tomography,” Journal of Nuclear Medicine, Vol. 33, No. 1, 1992, pp. 52-58.

[25] A. K. Mahapatra and R. Kamal, “A Textbook of Head
Injury,” Modern Publishers, Delhi, 1999.

[26] P. A. Hofman, S. Z. Stapert, M. J. Van Droonenbugh, et al., "MR Imaging, SPECT and Neurocognitive Peformance after Mild Traumatic Brain Injury," American Journal of Neuroradiology, Vol. 22, No. 3, 2001, pp. 441-449.

[27] A. N. Lassen and J. Astrup, "Cerbral Blood Flor: Normal Regulation and Ischemic Thresholds,” In: P. R. Weinstein and A. I. Faden, Eds., Protection of the Brain from Ischemia, Williams and Wilkins, Baltimore, 1990, pp. 7-19.

[28] N. K. Gowda, D. Agrawal, C. Bal, N. Chandrashekar, M. Tripati, G. P. Bandopadhyaya, A. Malhotra and A. K. Mahapatra, “Technetium Tc-99m Ethyl Cysteinate Dimer Brain Single-Photon Emission CT in Mild Traumatic Brain Injury: A Prospective Study," American Journal of Neuroradiology, Vol. 27, No. 2, 2006, pp. 447-451.

[29] M. Desco, J. López, C. Benito, A. Santos, P. Domínguez, S. Reig, C. Arango, P. García-Barreno, H. U. Lemke, M. W. Vannier, K. Inamura and A. G. Farman, "A Multimodality Workstation in Practice,” Computer Assisted Radiology and Surgery, 1999, pp. 218-222.

[30] J. López, M. Desco, C. Benito, A. Santos and P. GarcíaBarreno, "Estación de Tratamiento de Imagen Médica Multimodalidad,” Mapping, 1999. 